\title{
L'enseignement de la mécanique quantique grâce à un récit de fiction
}

Fabrice Ferlin, Philippe Lautesse, Juliette Tuaillon, Jean-Loup Héraud and Lionel Chaussard

\section{(2) OpenEdition}

1 Journals

\section{Electronic version}

URL: https://journals.openedition.org/ripes/3727

DOI: 10.4000/ripes.3727

ISSN: 2076-8427

\section{Publisher}

Association internationale de pédagogie universitaire

\section{Electronic reference}

Fabrice Ferlin, Philippe Lautesse, Juliette Tuaillon, Jean-Loup Héraud and Lionel Chaussard, "L'enseignement de la mécanique quantique grâce à un récit de fiction", Revue internationale de pédagogie de l'enseignement supérieur [Online], 37(3) | 2021, Online since 05 December 2021 connection on 27 September 2022. URL: http://journals.openedition.org/ripes/3727 ; DOI: https:// doi.org/10.4000/ripes.3727

This text was automatically generated on 27 September 2022

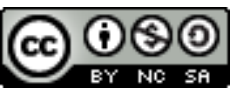

Creative Commons - Attribution-NonCommercial-ShareAlike 4.0 International - CC BY-NC-SA 4.0 https://creativecommons.org/licenses/by-nc-sa/4.0/ 


\section{L'enseignement de la mécanique quantique grâce à un récit de fiction}

Fabrice Ferlin, Philippe Lautesse, Juliette Tuaillon, Jean-Loup Héraud and Lionel Chaussard

\section{Introduction}

1 L'enseignement de la mécanique quantique (MQ), dont le formalisme mathématique est présenté en troisième année de licence de physique en France, est aussi, dans certaines universités, proposé dès la deuxième année avec une approche davantage qualitative, suivie d'exercices qui ne présentent pas de difficultés mathématiques supplémentaires par rapport à ceux d'autres parties du programme de physique (Ministère de l'Enseignement supérieur, de la Recherche et de l'Innovation, 2015). Une étude de Héraud, Lautesse, Ferlin et Chabot (2017) donne des exemples d'exercices proposés aux étudiants dans des introductions à la MQ faites dans l'enseignement secondaire en Terminale Scientifique (TS) et que l'on retrouve en partie en deuxième année de licence (L2). Cependant, il n'en est pas de même concernant les postures épistémologiques prises par les auteurs des manuels de TS, comme en atteste l'article de Lautesse, Vila Valls, Ferlin, Héraud et Chabot (2015). Ainsi, certains concepts mis en jeu en MQ tels que la nature de l'objet quantique et de la fonction d'onde, le problème de la mesure, les probabilités... peuvent s'avérer difficilement accessibles aux étudiants, comme le montrent les travaux de Ozcan (2011) et de Singh, Belloni et Christian (2006). Par ailleurs, l'interprétation de la MQ, qui est encore une question ouverte (Laloë, 2011), bien que le formalisme soit établi depuis maintenant près de 100 ans, est peu abordée dans l'enseignement supérieur en France. Or, l'enseignement de la MQ peut trouver sa pertinence (épistémologiquement parlant), en incluant les questions liées à ses interprétations (ontologiquement parlant) et en les confrontant à celles développées (souvent implicitement) par les étudiants, comme le souligne l'article de Baily et Finkelstein (2015). C'est dans ces perspectives à la fois épistémologiques et ontologiques que nous proposons une étude réflexive d'un roman de fiction réaliste, Isolation, de Greg Egan (2000), au travers de la spécificité du problème de la mesure. 
Nous faisons également l'hypothèse que proposer l'étude d'un tel roman est une source de motivation supplémentaire pour les étudiants, même si cette motivation ne présage en rien une meilleure compréhension de la MQ.

Le problème de la mesure est abordé dans le roman par l'intermédiaire de l'expérience de pensée du chat de Schrödinger dans laquelle la réduction du paquet d'ondes, et notamment sa cause, est discutée. En effet, d'où provient cette réduction : est-ce qu'un outil purement technologique suffit, faut-il que le signal soit enregistré sur un support afin qu'il soit par la suite observé par l'œil, doit-il être analysé par le cerveau?

Cette étude du phénomène de réduction du paquet d'ondes, auquel sont reliées les questions du choix de l'état final lors de cette réduction, revient à discuter des probabilités attachées aux états possibles d'un objet quantique avant la mesure proprement dite et plus généralement permet une réflexion sur l'ontologie de cet objet quantique et donc sur celle de la MQ.

Cet article est une réflexion épistémologique destinée aux enseignants de licence de physique, qui trouveront dans la section 5 , quelques pistes que nous leur soumettons. L'originalité de notre réflexion est d'aborder les questions listées ci-dessus par l'intermédiaire d'un roman de fiction et donc sous un angle différent, qui peut être complémentaire à un enseignement traditionnel. Nous postulons que l'utilisation d'un tel roman comme support de cours peut apporter aux étudiants une distance réflexive quant à la compréhension et à l'interprétation des phénomènes physiques mis en jeu. L'utilisation du roman de Egan dans l'enseignement de deuxième année de licence fera l'objet d'un prochain article.

5 Nous nous proposons donc d'aborder la problématique de la mesure, de discuter du rôle de l'observateur et de la spécificité des probabilités en $\mathrm{MQ}$, grâce au roman de Egan, Isolation (2000). Nous nous attacherons à montrer que ce roman peut être qualifié de " fiction réaliste ", telle qu'elle a été définie dans le cadre de la théorie de David Lewis (1973, 1978, 2007) dans l'article de Héraud, Lautesse, Ferlin et Chabot (2017). Dans un premier temps, dans son roman, Egan présente l'interprétation dite "standard $»^{1}$ de la mesure, celle qui est adoptée dans l'enseignement, au lycée et à l'université, en France comme dans bon nombre de pays dans le monde. Au fil de l'intrigue, l'auteur nous pousse à faire évoluer notre réflexion en faisant intervenir d'autres interprétations (avec ou sans réduction du paquet d'ondes) avec pour fil conducteur le problème de la mesure en physique quantique et la manipulation des probabilités quantiques qui constituent l'intrigue du roman. Cette manipulation fictionnelle, contrefactuelle avec notre monde quotidien, existe même pour des objets macroscopiques dans le roman de Egan.

6 Après la présentation de quatre des postulats de la MQ qui concernent notre étude et un rappel historique sur différentes approches de la problématique de la mesure en $\mathrm{MQ}$, nous présentons l'enseignement de la physique quantique au niveau de la deuxième année de licence en France. Nous expliquons pourquoi l'expérience de pensée du chat de Schrödinger, utilisée dans le récit de Egan, permet d'aborder cette problématique de la mesure. Nous voyons ensuite comment des extraits de ce roman peuvent être utilisés pour discuter, avec les étudiants de licence, du rôle de l'observateur et des probabilités en mécanique quantique. 


\section{La mesure en mécanique quantique : quelle problématisation?}

\subsection{Les postulats de la mécanique quantique, un problème de compatibilité?}

La mécanique quantique non relativiste repose sur un certain nombre de postulats. En fonction des besoins de notre étude, nous allons nous intéresser à quatre d'entre eux.

Le premier de ces postulats indique qu'à chaque instant $t$ fixé, l'état d'un système quantique est complètement défini par la donnée d'un vecteur d'état, noté $|\Psi(t)\rangle$. Ce vecteur d'état peut s'écrire comme une combinaison linéaire (ou superposition) d'états propres de l'opérateur correspondant à la grandeur physique mesurée.

Le deuxième postulat, dit de l'évolution, permet de caractériser l'évolution dans le temps du vecteur d'état d'un système quantique. Pour cela, Schrödinger a introduit une équation linéaire qui est vérifiée par ces vecteurs d'état $|\Psi(t)\rangle$ :

\section{$i \hbar \frac{d|\Psi(t)\rangle}{d t}=H|\Psi(t)\rangle$}

10 où $H$ est l'opérateur hamiltonien associé au système, c'est-à-dire un opérateur mathématique qui caractérise l'énergie du système quantique. On peut alors introduire le troisième postulat, dit de la mesure.

11 La mesure ou l'observation d'un système quantique, qui vise à connaître une grandeur physique liée à ce système, donne alors une valeur de cette grandeur qui est une valeur propre de l'opérateur correspondant à cette grandeur.

12 Ainsi, en mécanique quantique, une mesure fait passer le système d'un état de superposition (paquet d'ondes) à celui d'un état unique (vecteur propre de l'opérateur): on dit qu'il y a réduction du paquet d'ondes. Lors d'une mesure, l'évolution du vecteur d'état selon l'équation de Schrödinger est complètement rompue.

13 Le quatrième postulat nécessaire à notre étude est parfois appelé règle de Born (Laloë, 2011), ou principe de décomposition spectrale (Cohen Tannoudji, Diu et Laloë, 1977). Il concerne la question essentielle des probabilités en mécanique quantique.

14 Le vecteur d'état s'écrit alors comme une combinaison linéaire des vecteurs propres associés à des nombres complexes :

$$
|\Psi\rangle=\sum_{i=1}^{k} c_{i}\left|\Psi_{i}\right\rangle
$$

La mesure donnera la valeur propre de l'opérateur avec la probabilité $P\left(a_{n}\right)$ définie par :

$P\left(a_{n}\right)=\left|\left\langle\Psi_{n} \mid \Psi\right\rangle\right|^{2}=\left|c_{n}\right|^{2}$ 

différents pour l'évolution du même objet mathématique est une situation tout à fait inhabituelle en physique. Cette idée était d'ailleurs une totale nouveauté lorsqu'elle a été introduite, et reste actuellement unique dans toute la physique, ainsi qu'une source de difficultés - en particulier celles liées à la compatibilité entre les deux postulats.» (p. 41).

20 Cette coexistence surprenante des deux règles d'évolution correspond à ce que l'on appelle le problème de la mesure en mécanique quantique. Plusieurs théories, dont nous allons rappeler l'historique, ont été élaborées pour tenter d'expliquer ce qu'est la mesure en mécanique quantique et pour tenter de s'affranchir des difficultés liées à cette coexistence.

21 Ce problème de la mesure ouvre aussi sur des questions relatives à l'ontologie du vecteur d'état. Laloë (2011) souligne en effet :

22 «Beaucoup des difficultés rencontrées par ceux qui ont essayé (ou essayent toujours) de «vraiment comprendre» la mécanique quantique sont liées aux questions concernant le statut exact de $|\Psi(t)\rangle$. Le vecteur d'état décrit-il la réalité physique ellemême, ou seulement une connaissance (partielle) que nous aurions de cette réalité? Décrit-il seulement des ensembles de systèmes (description statistique), ou un système physique unique (une seule réalisation, un événement unique)? Si $|\Psi(t)\rangle$ contient une composante reliée à notre connaissance imparfaite de la réalité du système, n'est-il pas alors naturel de rechercher une description plus précise, qui devrait exister au moins en principe? Dans ce cas, quelle serait cette meilleure description de la réalité? » (p. xiv).

23 Laloë (2011) ajoute : « Sans aucun doute, le vecteur d'état est vraiment un curieux objet pour décrire la réalité! » (p. xv).

24 Ce vecteur d'état n'est en effet accessible qu'indirectement, par la mesure et l'observation, sans qu'on puisse en fait savoir ce qu'il était exactement avant l'observation, à cause de la réduction!

La problématique de l'enseignement de la mesure en L2 en mécanique quantique peut donc se résumer à la question que pose Laloë (2011) : « pourquoi donc attribuer aux mesures un caractère aussi particulier, de sorte que deux postulats distincts deviennent nécessaires?» (p. 42).

Pour mieux éclaircir cette problématique, nous présentons dans un premier temps les réponses historiques apportées.

\subsection{Réponses historiques}

Notons d'abord que dans l'interprétation de Copenhague au sens strict, développée notamment par Bohr, Heisenberg et Pauli, le problème de la mesure ne se pose pas. Cette interprétation correspond à une conception positiviste de la mécanique 
quantique, selon laquelle la théorie quantique ne décrit pas la réalité, mais permet seulement de connaître le résultat d'opérations de mesure. En physique quantique, le résultat de la mesure est unique, on ne doit pas s'interroger sur l'état d'un système avant cette mesure. La question d'une éventuelle contradiction entre une superposition d'états (avant la mesure) et l'état du système consécutif à la mesure ne se pose donc pas. Pour Bohr (1928) le postulat de la mesure s'applique dans le cas où l'on a un appareillage macroscopique qui obéit à la physique classique. Il n'y a pas de contradiction directe avec l'évolution de Schrödinger qui appartient au domaine où s'applique la physique quantique.

C'est von Neumann $(1932,1947)$ qui théorise pour la première fois le processus des mesures quantiques dans son livre de 1932. Pour lui, il n'y a qu'une seule physique et elle est quantique, et ce, que l'on soit dans le cas microscopique ou dans le cas macroscopique. Il étudie donc l'interaction entre le système mesuré et l'appareil de mesure qui forme une superposition d'états. Il montre que la superposition des états quantiques que l'on trouve au niveau du système mesuré doit se transférer à l'appareil de mesure. Ce dernier, bien que macroscopique, devrait être lui-même en état de superposition, si bien que, par exemple, l'aiguille d'un cadran indiquant le résultat d'une mesure devrait pointer à plusieurs endroits à la fois, ce qui n'est bien sûr pas ce que l'on observe. Ce problème ne pouvant être solutionné en mesurant l'appareil de mesure lui-même, ce qui ne ferait que déplacer le problème, la solution pourrait être de mettre un observateur conscient en bout de chaîne. Cet observateur romprait le cycle en réduisant le paquet d'ondes par un acte subjectif échappant à la physique. Cette interprétation de von Neumann (1932) sera particulièrement développée par London et Bauer (1939), puis Wigner (1967).

L'expérience de pensée du chat de Schrödinger, proposée en 1935, est une tentative pour répondre par l'absurde à la théorie de la mesure de von Neumann (1932). Nous développons cette expérience de pensée dans la sous-section 3.2., car celle-ci est utilisée, non seulement dans les enseignements de MQ, mais aussi dans le roman de Egan qui va nous servir de support de cours.

Une autre façon d'aborder le problème de la mesure, tout en restant dans des termes réalistes (par opposition à l'approche de von Neumann (1932) ou Wigner (1967)), est de lister trois propositions qui, considérées ensemble, posent un problème de compatibilité (Maudlin, 1995; Ruyant, 2016) :

(1) la fonction d'onde décrit entièrement l'état du système;

(2) la fonction d'onde évolue linéairement selon l'équation de Schrödinger;

(3) la mesure d'une propriété du système donne un seul résultat (réduction).

Si les deux premières propositions sont vraies, l'appareil de mesure devrait se retrouver en état de superposition, ce qui est en contradiction avec la troisième proposition.

Il faut donc faire un choix de deux des trois propositions. Selon le choix effectué, on aura différentes interprétations de la MQ. Rejeter la première proposition conduit à des théories à variables cachées, dont l'exemple le plus connu est la théorie de Bohm (1952), dans laquelle on retrouve des trajectoires de type classique pour les objets quantiques. Rejeter la deuxième proposition conduit à modifier l'évolution de type Schrödinger par une évolution de type stochastique. Un modèle de cette approche est le modèle dit GRW, du nom des trois physiciens Ghirardi, Rimini, Weber, qui ont 
développé cette théorie (Ghirardi, Rimini et Weber, 1985, 1986), où l'on rajoute des termes stochastiques non linéaires à l'équation de Schrödinger. Enfin, on peut rejeter la troisième proposition, comme c'est le cas de la théorie des états relatifs (Everett, 1957), que DeWitt reformulera en termes de multivers (DeWitt et Graham, 1973). Dans ce cas, on n'ajoute rien au formalisme quantique et on considère au contraire qu'il décrit totalement la réalité. Il y a superposition des états, chaque état est réel et tous les résultats possibles sont réalisés lors de la mesure, ce qui entraine un découpage de l'univers en autant de branches parallèles. Dans ce cas, le postulat de réduction du paquet d'ondes n'a pas lieu d'être, il n'y a pas de réduction et la seule règle d'évolution est donnée par l'équation de Schrödinger.

Une autre approche qui correspond à un rejet de la troisième proposition est celle de la décohérence (Zeh, 1970). Selon cette théorie, la disparition des superpositions quantiques dans le cas des objets macroscopiques s'explique par les interactions de ces objets avec leur environnement. La réduction du paquet d'ondes est alors un processus très rapide, obéissant à la dynamique de l'équation de Schrödinger, rendant inutile le postulat de réduction.

Ce questionnement sur la mesure quantique reste une préoccupation constante dans l'histoire conceptuelle de la MQ. Certes, l'enseignement de la MQ, dans le cadre d'une prise de parti didactique mathématique et formelle, peut faire l'économie d'une telle interrogation épistémologique, mais celle-ci n'en restera pas moins un point aveugle de la théorie.

Nous allons maintenant décrire ce qui est enseigné dans les premières années d'enseignement supérieur en France.

\section{La mesure dans l'enseignement de la physique quantique en L2 en France}

\subsection{L'enseignement de la physique quantique en L2 en France}

Dans l'enseignement universitaire, la physique moderne est enseignée sans interruption depuis le début des années 1920, donc très peu de temps après les découvertes majeures dans le domaine. A l'heure actuelle, les enseignants du supérieur utilisent «le référentiel de compétences de Licence» qui définit des «objectifs de formation ». Au sein de ce référentiel, la $\mathrm{MQ}$ est incluse dans un référentiel plus spécifique intitulé «manipuler les mécanismes fondamentaux à l'échelle microscopique, modéliser les phénomènes macroscopiques, relier un phénomène macroscopique aux processus microscopiques ". Ce référentiel indique aussi qu'une liberté est laissée aux enseignants dans leur façon d'aborder le sujet :

«Il appartient aux établissements de préciser, décliner ou compléter ces référentiels lors de l'élaboration des parcours types. Mais il appartient surtout aux équipes, dans le respect de l'autonomie des établissements et des dynamiques déjà enclenchées, d'organiser les modalités de construction des compétences figurant au référentiel » (Ministère de l'Enseignement supérieur, de la Recherche et de l'Innovation, 2015).

41 Cette liberté pédagogique se retrouve effectivement dans les universités françaises où une introduction à la physique dite moderne est parfois dispensée dès la deuxième année de licence (L2) afin de permettre aux étudiants de mieux appréhender les 
nouveaux concepts qu'impose l'apprentissage de la mécanique quantique. Là encore, on note une grande variété d'approches dans l'enseignement dispensé en L2. Une consultation des sites internet d'une cinquantaine d'universités françaises montre qu'une quinzaine d'entre elles mentionnent dans leur onglet « offre de formation » ou équivalent, qu'une "introduction à la mécanique quantique» est proposée en deuxième année de licence. Les programmes ne sont pas toujours détaillés, mais lorsqu'ils le sont, on constate qu'ils varient dans leur contenu, mais aussi dans leur approche, tant pédagogique qu'épistémologique, de la discipline. Cependant, la consultation de ces sites montre que les programmes restent le plus souvent basés sur la réflexion et la prise de conscience des nouvelles notions contre-intuitives de la mécanique quantique, plutôt que sur les concepts mathématiques.

Cette disparité dans la façon d'aborder cette discipline se retrouve dans les livres disponibles dans les bibliothèques universitaires françaises. L'expérience de pensée du chat de Schrödinger, mais aussi celle des fentes de Young par exemple, sont fréquemment utilisées pour introduire les notions de superposition d'états et de mesure, avec des approches très différentes suivant les auteurs. Pour certains auteurs, tels que Feynman (1979), l'expérience des fentes de Young est expliquée sans engager le formalisme mathématique complexe nécessaire à la mécanique quantique et favorise donc une approche réflexive de cet enseignement. De même, Lévy-Leblond (1984), dans son ouvrage Quantique - Rudiments, propose une approche réflexive de la mécanique quantique. En revanche, des auteurs tels que Le Bellac (2007), dans son livre Physique quantique, Cohen-Tannoudji et al. (1997), dans leur livre Mécanique quantique, ou encore Basdevant (2017), dans son livre Introduction à la physique quantique, exploitent ces expériences en utilisant la notion de vecteur d'état, qui nécessite au préalable une maitrise du langage mathématique afin de comprendre la portée de ces expériences. D'autres encore écrivent même qu'une réflexion de ce type n'est pas nécessaire et le précisent dans leur avant-propos comme dans le livre Physique quantique: introduction de Ngô et Ngô (1991) :

«Ce livre étant une introduction à la mécanique quantique, nous avons adopté le point de vue dit de "l'Ecole de Copenhague", ce qui signifie que nous n'avons pas évoqué certains problèmes liés à son interprétation. Ces derniers sont d'ailleurs peu importants pour ce qui concerne l'utilisation pratique de la mécanique quantique.» (p. XIV)

\subsection{L'expérience de pensée du chat de Schrödinger}

44 L'expérience de pensée du chat de Schrödinger nous permet d'aborder les questions que nous développons dans cet article, telles que la mesure, les probabilités et enfin le rôle de l'observateur. Commençons par expliquer en quoi consiste cette expérience de pensée.

Un chat est enfermé dans une boîte dans laquelle se trouve une fiole contenant un gaz mortel. Cette fiole se cassera et libérera son gaz mortel lorsqu'elle sera touchée par une particule émise lors de la désintégration d'un atome radioactif, ce qui entraîne la mort $\mathrm{du}$ chat. Pour pouvoir faire un parallèle entre cette expérience et la mécanique quantique, le chat doit être considéré comme ayant le même comportement que les objets quantiques. Le chat est donc représenté par un vecteur d'état $|\Psi(t)\rangle$. Il est alors possible d'utiliser les notions de mesure, de probabilités et du rôle de l'observateur 
pour ce chat quantique. La notion de probabilité est ici introduite par la nature probabiliste de la désintégration de cet atome radioactif. Le problème de la mesure est introduit car le chat peut être considéré comme un instrument de mesure puisqu'il nous indique si cet atome s'est désintégré ou non. Le rôle de l'observateur est introduit, car la réduction du paquet d'ondes, donc la mesure de l'état du chat, mort ou vivant, peut se faire à plusieurs niveaux, lorsque l'on ouvre la boîte, lorsque l'on sait que l'atome radioactif s'est désintégré. La question centrale de la mesure d'un objet quantique apparait alors brutalement. L'observation du chat par un détecteur, donc la détermination de son état, modifie le résultat de l'expérience. Chaque objet quantique est une superposition d'états, un paquet d'ondes, qui reste à l'état de superposition tant qu'il n'a pas été perturbé, détecté, mesuré. Avant la mesure, le chat est une superposition d'états «chat mort » et «chat vivant » dont la probabilité attachée à chacun de ces états va évoluer tout au long de l'expérience. La notion de probabilité est donc intrinsèquement liée aux objets quantiques par l'intermédiaire du vecteur d'état. Le chat quantique est alors représenté par le vecteur d'état:

$|\Psi(t)\rangle=c_{\text {mort }}(t)\left|\Psi_{\text {mort }}\right\rangle+c_{\text {vivant }}(t)\left|\Psi_{\text {vivant }}\right\rangle$

où $c_{\text {mort }}$ et $c_{\text {vivant }}$ sont des nombres complexes, dont la norme au carré représente la probabilité de trouver le chat dans un de ces deux états. On peut alors écrire :

$|\Psi(t)|^{2}=\left|c_{\text {mort }}(t)\right|^{2}+\left|c_{\text {vivant }}(t)\right|^{2}=1$

49 Au début de l'expérience la probabilité attachée à l'état "vivant » est proche de « 1 » alors que celle attachée à l'état «mort » est proche de « 0 ». Au cours du temps, tant qu'il n'y a pas d'acte de mesure, ces valeurs vont progressivement évoluer, jusqu'à ce que la probabilité attachée à l'état « vivant » devienne pratiquement nulle et que celle attachée à l'état «mort» se rapproche très fortement de " 1 », au même titre que l'évolution de l'état de l'atome radioactif.

Lors d'une mesure, comment s'impose une probabilité de " 1 » à l'un de ces deux états et nécessairement de « 0 » à l'autre? Quel va être l'élément déclencheur de la réduction du paquet d'ondes du chat? Est-ce qu'il va falloir nécessairement ouvrir la boîte? Une fois ouverte, il faut se poser la question du rôle de l'observateur. Est-il nécessaire qu'il y ait un observateur, voire une conscience, à l'ouverture de cette boîte, ou est-ce qu'un instrument technologique peut suffire à réduire le paquet d'ondes du chat, sans qu'aucune conscience ne soit là pour le voir? Une autre solution est peut-être envisageable. Tout ne se passerait-il pas dans la boîte, sans qu'il n'y ait besoin de l'ouvrir? Et la désintégration de l'atome ne réduit-elle pas instantanément le paquet d'ondes du chat, sans qu'aucun moyen de le détecter ne soit présent dans la boite ni visible de l'extérieur? Cette expérience pose aussi la question des superpositions d'états pour des objets macroscopiques, comme un chat, superpositions conformes à l'équation de Schrödinger, mais que l'on n'observe pas dans la vie courante. Ces questions n'ont pas de réponse toute faite. Chaque interprétation de la mécanique quantique que l'on a décrite précédemment apporte une réponse différente.

Alors que ces questions ne sont que très rarement abordées dans les ouvrages d'enseignement, nous avons sélectionné un support didactique que nous estimons approprié : le roman de Egan où ces expériences sont utilisées, ce qui va nous permettre d'illustrer les problèmes liés à la mesure, aux probabilités et au rôle de l'observateur. 


\section{Comment aborder le problème de la mesure? Sélection d'un récit de fiction}

\subsection{Un roman épistémologique : le recours à la physique quantique pour résoudre l'énigme du récit}

Dans le roman de Egan, qui se situe dans un futur proche (entre 2030 et 2080), la planète Terre, sur laquelle vivent les personnages, et son système solaire sont isolés du reste de l'univers par une «bulle », créée par des entités extra-terrestres (d'où le titre du roman : Isolation).

3 La Terre est en effet un endroit atypique de l'Univers où a constamment lieu le phénomène quantique que l'on appelle réduction du paquet d'ondes. Cette réduction est générée par le cerveau des êtres humains et des animaux supérieurs lors de leurs observations et semble résulter d'une mutation génétique ayant eu lieu chez un mammifère dans une lointaine préhistoire. Ailleurs dans l'Univers, ce phénomène de réduction n'existe pas et on se retrouve donc dans une situation similaire à la théorie des multivers de DeWitt (1973).

Ainsi, les humains détruisent tous les états de ce qu'ils observent, sauf l'état propre résultant de la réduction. Cette réduction "détruit» donc, par l'observation, une infinité de mondes possibles. Ceci a été perçu comme une menace par des civilisations extra-terrestres qui ont décidé d'isoler la Terre à l'intérieur d'une bulle protectrice, empêchant toute observation de l'univers lointain par les terriens.

5 Sur Terre, une "handicapée mentale», Laura Andrews, arrive mystérieusement à s'échapper de l'institut spécialisé où elle est enfermée. Un détective, Nick, héros principal du roman et narrateur, est chargé par un mystérieux commanditaire de retrouver Laura. Arrivé pratiquement au but, sur le point d'emmener Laura de là où elle était à nouveau enfermée, il est capturé par les services de sécurité, puis est embauché par ceux qui détiennent Laura, comme agent de sécurité. Ses fonctions consisteront bientôt à assurer la sécurité d'une physicienne, Po-kwai, qui effectue une expérience de mécanique quantique de type Stern et Gerlach ${ }^{2}$. On se rend bientôt compte que le but de cette expérience est d'arriver à contrôler les probabilités d'obtenir l'une des deux valeurs du spin des atomes d'argent.

6 Le narrateur comprend bientôt que cette faculté de modifier les probabilités et de rendre certains états totalement improbables constitue la base des pouvoirs d'évasion de Laura et que ses nouveaux employeurs, dans le plus grand secret, essayent de se rendre maîtres de ce pouvoir. A cette fin, ils sont parvenus à créer un outil, appelé «mod», implanté dans le cerveau de la physicienne Po-kwai, qui doit lui permettre d'une part d'empêcher les processus de réduction par le cerveau et d'autre part d'être capable de modifier la probabilité des différents états quantiques constituant l'état superposé avant la mesure et donc de choisir l'état final, après avoir enfin déclenché la réduction. Les enjeux financiers et de pouvoir sont considérables, d'où un secret renforcé et des mesures draconiennes de sécurité. Le narrateur va lui-même bientôt être engagé dans un complot visant à voler ce nouveau «mod» révolutionnaire, nommé « Ensemble ».

Le narrateur, pendant le sommeil de Po-kwai, parvient à utiliser le " mod », d'où l'accès à des capacités hors du commun qui vont lui permettre de s'introduire sans difficultés 
dans les lieux les plus sécurisés. A la fin du roman, un état de non-réduction, dit d'étalement ${ }^{3}$, s'instaure sur la ville où se déroule l'intrigue, avec des effets spectaculaires et tragiques. roman, le monde sans réduction ainsi généré est alors un monde possible le plus proche du monde actuel. scientifiquement. Ainsi, les lois de la physique du monde possible créé par Egan forment un ensemble cohérent.

\subsection{Le choix du roman Isolation : une problématisation épistémologique de la physique quantique dans l'ontologie d'un « monde possible »?}

Héraud et al. (2017) ont défini, sur la base de la théorie de Lewis, les critères auxquels doit satisfaire un récit de fiction pour qu'il puisse engager une problématisation épistémologique, portant dans le cas considéré sur la nature des concepts contreintuitifs de physique quantique dans un monde possible.

L'intérêt didactique réside pour nous dans cette décentration non arbitraire des lois quantiques invisibles dans notre monde sur un monde possible auquel le comparer.

Nous reprenons ci-dessous les cinq critères définis dans l'article de Héraud et al. (2017) pour attester la pertinence épistémologique du roman de fiction Isolation.

- Principe de contrefactualité

Le principe de contrefactualité, fondateur des mondes possibles, s'énonce dans une proposition qui, pour un fait (ou une loi) contraire au monde actuel, donc faux dans notre monde, établit une conséquence vraie dans un autre monde, appelé monde possible. Dans le roman, l'hypothèse contrefactuelle d'une observation non réductrice, entraîne comme conséquence la construction d'un monde physique qui n'est pas soumis au principe de réduction du paquet d'ondes, ce qui permet par la suite la manipulation des probabilités quantiques.

- Principe de similarité comparative

- Principe de consistance

Ce principe consiste à proposer, dans un récit, une version fictionnelle consistante - Principe d'accessibilité cognitive 

un lecteur. Ainsi, le roman de Egan, à partir de la non-réduction du paquet d'ondes et de la manipulation des probabilités permet de rendre visibles, sur un registre macroscopique, des phénomènes quantiques invisibles dans notre monde actuel.

Ce principe impose de pouvoir reconstruire les lois du monde actuel par confrontation avec les faits du monde possible. Dans le cas du roman de Egan, le principe de contrôle de la réduction du paquet d'ondes, discuté dans le monde contrefactuel du récit, permet au lecteur de mieux s'approprier le phénomène de la mesure dans la physique quantique.

Nous voyons donc que le roman Isolation satisfait aux cinq critères rappelés ci-dessus. En effet, l'introduction progressive au fil du roman des concepts de la mécanique quantique (probabilités, superposition, réduction et rôle de l'observateur) permet de proposer une approche réflexive aux étudiants, concernant ces différentes questions. Nous pouvons maintenant proposer une lecture épistémologique du texte dans le but d'en faire un outil didactique.

\section{Enjeux épistémologiques du roman de Egan pour l'enseignement de L2}

Pour notre analyse, nous avons regroupé les citations du roman de Egan en trois soussections. Dans la première sous-section, nous analysons le problème de la mesure et du rôle de l'observateur en mécanique quantique, qui est posé très clairement, au travers d'une discussion entre la physicienne Po-kwai et le détective Nick, sur l'expérience du chat de Schrödinger. Dans la deuxième sous-section, les interprétations et/ou les théories alternatives à l'interprétation standard sont abordées grâce à leur développement et plus particulièrement la théorie des états relatifs de Everett (1957) et son extension aux multivers formulée par DeWitt (1973). La troisième sous-section propose de voir comment un monde contrefactuel est construit avec deux principes forts que sont la non-réduction du paquet d'ondes et le contrôle des probabilités. Pour cela, nous utilisons des extraits portant sur l'apparition de phénomènes extraordinaires engendrés par l'étalement (superposition) des phénomènes possibles et la manipulation des probabilités.

79 Le choix des extraits est guidé par leur future utilisation lors de cours dispensés en L2 à l'Université de Lyon. Le but est d'utiliser un monde contrefactuel fondé sur une autre interprétation de la MQ que l'interprétation standard généralement proposée aux étudiants. Plus généralement, cette stratégie permettrait aux étudiants de s'approprier, par une approche réflexive complémentaire de l'enseignement du formalisme mathématique, les fondements de la physique quantique.

\subsection{La problématique de la mesure dans le roman de Egan}

80 Le roman de Egan offre des passages didactiques concernant la problématique de la mesure. Il utilise notamment l'expérience de pensée du chat de Schrödinger comme introduction aux problèmes posés par la mesure et la superposition des états qui forment le paquet d'ondes :

Revue internationale de pédagogie de l'enseignement supérieur, 37(3) | 2021 
81 «Cela mériterait de faire la une de Nature demain : "LE RÔLE DE L'OBSERVATEUR DANS LA MECANIQUE QUANTIQUE CONFIRMÉ - AVEC VARIATIONS!" [...] Avez-vous entendu parler du problème de la mesure quantique?

82 - Non.

83 - Et du chat de Schrödinger?

84 - Bien sûr.

85 - Eh bien, le chat de Schrödinger est une illustration du problème de la mesure quantique. La mécanique quantique décrit des systèmes microscopiques - des particules subatomiques, des atomes, des molécules - à l'aide d'un formalisme mathématique appelé fonction d'onde. A partir de la fonction d'onde, on peut prévoir les probabilités d'obtenir divers résultats quand on effectue des mesures sur le système. $[\ldots]$

86 - En voyant dans quel état il se trouve [le chat], on mesure si l'atome s'est ou non désintégré.

87 - Et alors... où est le problème?

88 - Le problème, c'est qu'avant la mesure, la fonction d'onde ne vous dit pas ce que va être le résultat; elle vous dit seulement que chacun d'eux a une chance sur deux ${ }^{4}$ de se réaliser. Mais une fois que vous avez fait la mesure, une deuxième mesure sur le même système donnera toujours le même résultat [...] En termes de fonction d'onde, le fait d'effectuer la mesure a - d'une certaine manière - transformé un mélange de deux ondes, représentant les deux possibilités, en une onde "pure" - qu'on appelle un état propre - qui n'en représente qu'une. C'est ce qu'on appelle la "réduction du paquet d'ondes". » (p. 172-175).

89 Le texte expose un grand nombre de concepts de la mécanique quantique, impliqués dans l'interprétation standard, dont la critique est visée par Schrödinger dans son expérience de pensée que nous avons décrite en détails dans la sous-section 3.2. On constate que l'auteur, par l'intermédiaire de Nick, ne compte pas nous faire accepter aussi facilement ce résultat. Egan pose des questions liées à la mesure en mécanique quantique :

90 «Mais pourquoi une mesure devrait être spéciale? Pourquoi devrait-elle réduire le paquet d'ondes? Pourquoi un quelconque instrument de mesure - lui-même composé d'atomes individuels [...] - causerait-il la réduction d'un mélange de possibilités à une et une seule d'entre elles?» (p. 175).

91 Le roman, par l'intermédiaire de la physicienne Po-kwai, expose nettement les enjeux du problème de la mesure, évoquant les problèmes liés à la chaîne de von Neumann (1932) et à l'extension des superpositions au niveau macroscopique, si bien que le novice qu'est Nick est amené, comme peut-être le lecteur - et donc l'étudiant -, à se poser la question de la pertinence même de la théorie quantique.

92 "le chat [doit] se résumer au mélange de l'état "mort" et de l'état "vivant". Alors pourquoi voyons-nous donc toujours le chat dans un état pur, mort ou vivant?

93 - Peut-être que la théorie est tout simplement fausse.

94 - Non, ce n'est pas aussi facile que ça. Jamais une théorie scientifique n'a rencontré autant de succès que la mécanique quantique - sous réserve d'accepter la réduction du paquet d'ondes. » (p. 175-176). 

physique quantique. Comme pour les notions introduites précédemment, l'exposé de ces diverses interprétations est suffisamment clair pour être utilisé avec les étudiants de L2.

\subsubsection{L'interprétation standard}


Une troisième interprétation correspond à des explications développées entre autres par Penrose et par le groupe GRW (Ghirardi, Rimini et Weber, 1985, 1986). Elles sousentendent que la théorie standard n'est pas complète, ce qui nécessite l'introduction de variables cachées ou de termes supplémentaires dans l'équation de Schrödinger afin de répondre à son incomplétude : «Certains ont invoqué des effets thermodynamiques, la gravité quantique, des non-linéarités hypothétiques dans les équations... toutes sortes de choses. Qui n'expliquent jamais complètement les faits. » (p. 177).

Un autre extrait évoque des types d'interprétations sans réduction :

"J'ai lu un article dans Physical Review ce matin: un tout nouveau traitement du problème de la mesure. Ils rajoutent quelques dimensions supplémentaires à l'espacetemps; introduisent quelques non-linéarités, quelques asymétries, et divers facteurs pour noyer le poisson; et - miracle des miracles! - la réduction du paquet d'ondes disparaît. » (p. 298).

106 La quatrième est celle dite des univers multiples, où précisément la réduction du paquet d'ondes n'a pas lieu. Tous les mondes restent présents, malgré la mesure :

107 «Et puis il y a la théorie des univers multiples...

108 - Des histoires alternatives, des univers parallèles...

109 - Exactement. Dans la théorie des univers multiples, le paquet d'ondes n'est pas réduit. C'est tout l'univers qui se scinde en versions différentes, une pour chaque mesure possible. Dans un univers, le chat est mort et un expérimentateur a constaté son décès; dans un autre univers, il est vivant et c'est bien ce que l'observateur a vu.» (p. 177).

110 L'intrigue nous amène à concevoir les conséquences au plan de la conscience d'un univers sans réduction avec l'extrait suivant, dans lequel l'interprétation des multivers est opposée à un autre univers sans réduction, où les différents états coexistent au lieu d'être chacun dans des branches séparées de l'univers :

111 «La planète entière étalée, définitivement... qu'éprouveraient exactement les gens? Rien - parce que aucune réduction ne rendrait quoi que ce soit réel? [...] Tout, séparément - une conscience isolée par état propre, comme dans le modèle des univers multiples? Ou tout, simultanément - une cacophonie de possibilités en surimpression? Ce que j'ai moi-même vécu - ou du moins le souvenir qui m'en reste après la réduction - ne reflète peut-être rien de la nature de ce qui se passerait en l'absence de toute réduction future. $\mathrm{Si}$ plus rien ne forçait le passé à être unique, notre perception des choses pourrait en être radicalement modifiée. [...] J'espère seulement que mon moi étalé est d'accord.» (p. 344).

112 La suite de l'intrigue montrera que cette dernière interprétation est celle qui correspond à la physique du monde contrefactuel développée dans le roman. Seule la réduction opérée constamment par les cerveaux humains empêche ces spectaculaires superpositions macroscopiques de se manifester sur la planète Terre.

\subsubsection{Une discussion sur la réduction du paquet d'ondes}

113 Dans le roman de Egan intervient alors une critique de l'interprétation des multivers.

114 «L'ennui, c'est que la théorie ne dit pas pourquoi cela devrait se passer ainsi - ou même à quel instant l'univers se scinde : détecteur? bouteille? chat? humain? Elle ne donne pas vraiment de réponse. 
115 - Peut-être qu'il n'y a pas de réponse; peut-être que tout cela n'est que du pinaillage métaphysique... » (p. 177). "Il reste quoi qu'il arrive une question essentielle en $\mathrm{MQ}$, si la réduction a bien lieu, à quel niveau se produit-elle? "- Elle attend patiemment que je comprenne - avec un sourire vaguement satisfait.

Dans le cerveau? D'une certaine manière, vous avez montré que la réduction du paquet d'ondes se passe dans le cerveau?

118 - Oui." » (p. 177-178).

119 Dans le roman, Egan considère donc que l'acte de réduction se situe dans le cerveau, mais il est tout de même permis de douter de cela :

120 «C'est une très vieille idée; un grand nombre des pionniers de la mécanique quantique croyaient que l'observateur jouait un rôle crucial - qu'il ne suffisait pas seulement d'un appareil de mesure pour réduire le paquet d'ondes. Mais ça a pris plus d'un siècle pour repérer exactement où cela se passe dans l'observateur. Je ne sais toujours pas si je dois croire le moindre mot de tout cela » (p. 181).

121 Il est possible, à ce stade de la lecture, que l'enseignant fasse participer les étudiants afin qu'ils s'interrogent sur le problème de la réduction du paquet d'ondes et sur ce qui le réduit le cas échéant. Un détecteur suffit-il ou faut-il un organisme vivant? Si c'est le cas, est-il nécessaire que ce soit un être humain ou un simple chat ferait-il l'affaire? Les étudiants pourraient caractériser ce qui est cohérent avec l'interprétation standard, développée dans le cours, et ce qui ne l'est pas.

\subsection{Qu'est-ce qu'un monde sous contrôle des probabilités peut nous apprendre sur notre monde quotidien?}

Nous allons voir maintenant comment le récit construit un monde contrefactuel en permettant à certains personnages du roman de contrôler les probabilités attachées à chaque état possible. Cette hypothèse est inévitablement attachée à la superposition des états possibles avant la mesure; il faut nécessairement être conscient d'avoir un choix avant la mesure pour pouvoir en faire un. Ces extraits vont montrer que la superposition des états avant la mesure, puis progressivement la possibilité offerte aux personnages d'influencer le choix du résultat, sont, non seulement tout à fait compatibles avec l'intrigue et le monde contrefactuel proposé dans ce roman, mais répondent aussi à la question centrale du roman. En effet, comment Laura, présentée comme "handicapée cérébrale», incapable de se mouvoir et de prendre une quelconque décision, arrive-t-elle à s'échapper de l'institut dans lequel elle est enfermée?

De nombreux extraits mettent les personnages dans des situations de superposition, soit pour eux, soit pour les personnes qui les entourent. On lit par exemple qu'une femme, morte plusieurs années auparavant, réapparaît à son mari, Nick : «Je lâche sa main et elle... ... remplit la pièce.

Elle s'étend, s'étale, se reproduit, comme une boîte de couleurs holographique devenue folle. Je me lève brutalement, renversant la chaise, tandis que l'espace qui m'entoure 
s'encombre d'un nombre croissant d'exemplaires de son corps illusoire. Je me voile le visage, mais je peux toujours la sentir, qui me frôle de tous côtés. » (p. 168).

Ces exemples de superposition se trouvent, la plupart du temps, attachés au phénomène de réduction du paquet d'ondes. Comme nous l'avons vu, la question est celle de savoir où, quand, à quel niveau de la mesure se produit cette réduction. On voit clairement l'aboutissement de leurs réflexions lorsque Nick pose cette question à Pokwai :

«D'une certaine manière, vous avez montré que la réduction du paquet d'ondes se passe dans le cerveau?

129 - Oui.

130 - Mais... comment? [...]

131 - Le mod fait deux choses. La première, c'est qu'il m'empêche de réduire le paquet d'ondes; il met hors service les parties du cerveau qui font ça en temps normal. [...]

- Mais le mod me permet aussi de manipuler les états propres - maintenant que je ne les détruis plus tous sauf un, maladroitement et aléatoirement. Il me laisse changer leurs forces relatives - et donc modifier les probabilités des résultats possibles de l'expérience. » (p. 178).

La contrefactualité introduite dans le roman apparaît alors clairement. A la réduction du paquet d'ondes s'ajoute la possibilité de choisir le résultat de cette réduction, et ce, grâce au cerveau. Mais ces facultés ne sont pas accessibles à tous; il faut posséder le «mod». Les personnes qui l'ont ont accès aux états superposés, c'est-à-dire à la fonction d'onde qui caractérise l'état du système avant la mesure, donc à l'état des objets avant la mesure, ce qui est impossible dans notre monde actuel. Un autre monde est alors possible. Les personnes possédant ce « mod » ont en plus la capacité de choisir leur destin en contrôlant les probabilités, ils peuvent choisir le résultat de la mesure.

On découvre que la physicienne, qui sert de cobaye dans une expérience qui permet de déterminer le spin des atomes (dans l'expérience de Stern et Gerlach), est bien dotée de ce «mod». Elle choisit l'état des atomes avant que ceux-ci soient observés par un détecteur. L'influence du «mod» connecté au cerveau de Po-kwai déjoue toutes les probabilités. Nick, découvrant cette capacité, pressent que le monde qui pourrait ainsi émerger serait fascinant: «Si Chung Po-kwai peut franchir des portes fermées en dormant... vous imaginez ce que pourrait réaliser un utilisateur expérimenté, entièrement conscient du potentiel du mod? » (p. 227).

Nick se rend progressivement compte qu'il possède, lui aussi, une partie de ce « mod", lors du sommeil de Po-kwai. Pour s'en convaincre, il joue aux dés et constate très vite qu'un enchaînement de résultats, de succession de paires d'as, se produit, bien au-delà que ce qu'offrent les probabilités du monde actuel. Il possède donc les mêmes facultés que Po-kwai : "Je sors le générateur de dés, invoque les mods et me mets au travail. » (p. 254).

Po-kwai avait raison : c'est mon moi étalé qui fait tout le travail.

«Je vais devoir accepter la vérité toute nue : je suis en train de vivre des événements qui seront (ou ont été) arrachés à un milliard de milliards de possibilités par l'effort collectif de quelques milliards de milliards de versions de moi-même... dont je suis sur le point de massacrer la plupart (à moins que je ne l'aie déjà fait). » (p. 257). 

Néanmoins, cette fiction réaliste peut être une ressource extrêmement intéressante pour des étudiants de licence de physique, que ce soit pour discuter la problématique de la mesure en physique quantique, des interprétations de la mécanique quantique, du rôle potentiel de la conscience dans cette théorie ou encore des probabilités quantiques. Au final, des étudiants de licence pourraient utiliser le caractère contrefactuel, lié à la manipulation des probabilités et au rôle de la conscience pour discuter l'interprétation de la fonction d'onde (dans l'équation de Schrödinger) : est-ce un objet sans ontologie ou caractérise-t-elle au contraire l'état du système et dans ce cas quel est le rôle de la conscience (Laloë, 2011)? 


\section{Conclusion et perspectives}

Notre article part de la constatation que la mécanique quantique est abordée davantage conceptuellement en deuxième année d'université en France, alors que l'aspect formalisme mathématique (satisfaisant à l'interprétation standard) prédomine dans la troisième année d'enseignement. De notre point de vue, les deux aspects sont complémentaires et nous proposons d'introduire un questionnement épistémologique sur les concepts physiques en jeu par l'intermédiaire du roman de fiction réaliste (qui satisfait à la théorie des mondes possibles de Lewis), Isolation, de Egan.

Notre choix d'interroger le statut particulier de la mesure en mécanique quantique se justifie dans ce contexte. Ce choix oblige, en effet, à déplacer la réflexion et à la porter sur les relations entre les différents concepts impliqués dans la réduction lors d'une mesure : états de superposition, probabilités des résultats, et rôle de l'observateur. Nous avons voulu ouvrir cette question encore controversée aujourd'hui et peu enseignée.

Dans la perspective d'une application didactique, le récit de fiction choisi, Isolation, est propre à stimuler et à engager une telle réflexion pour les enseignants, comme pour les étudiants. Cette réflexion est en lien avec un triple enjeu éducatif. Le premier consiste à opérer une prise de recul épistémologique par rapport aux contenus des enseignements. Le deuxième consiste à "transposer ", dans le contexte ontologique d'un monde possible, les concepts de la mécanique quantique pour reconfigurer ce monde de façon rigoureuse, de manière à bien mettre en évidence les problèmes liés à la mesure et aux probabilités. Le troisième consiste à introduire une dimension culturelle critique dans la science, notamment pour les futurs chercheurs et/ou enseignants.

151 Cette étude est une première étape indispensable dans la perspective d'intégrer, dans une seconde étape des situations didactiques, pour l'enseignement, avec l'objectif d'aménager un espace de discussion épistémologique portant sur les trois enjeux énoncés ci-dessus. Différentes activités pourront être conçues et réalisées par les enseignants de la deuxième année de licence de physique. Nous proposons d'utiliser des extraits de Isolation pour analyser en cours l'expérience de pensée du chat de Schrödinger abordée dans le roman. En parallèle, des groupes d'étudiants pourront produire des vidéos pédagogiques relatives aux concepts abordés à partir d'extraits de Isolation. Ces pistes, évoquées ici, seront discutées dans un prochain article.

152 La question sera alors de confronter la pertinence de notre travail préparatoire avec la validité opératoire d'une démarche d'éducation épistémologique. En proposant de construire et de réaliser des temps de réflexions conceptuelles, notre objectif est double : il vise à procurer aux étudiants une conscience à la fois réflexive et critique sur l'objet enseigné (prochain article) en fournissant aux enseignants des exemples de supports permettant d'aboutir à l'exigence d'une réflexion épistémologique (article actuel). 


\section{BIBLIOGRAPHY}

Baily, C. et Finkelstein, N.D. (2015). Teaching quantum interpretations: Revisiting the goals and practices of introductory quantum physics courses. Physical Review Special Topics - Physics Education Research, 11, 020124.

Basdevant, J. L. (2017). Introduction à la physique quantique. De Boeck Supérieur.

Bohm, D. (1952). A suggested Interpretation of the Quantum Theory in Terms of 'Hidden' Variables I. Physical Review, 85(2), 166-179.

Bohr, N. (1928). The quantum postulate and the recent development of atomic theory, Nature, $521,580-590$.

Cohen-Tannoudji, C., Diu, B. et Laloë, F. (1977). Mécanique Quantique. Hermann.

DeWitt, B. S. et Graham, N. (dir.), (1973). The Many-Worlds Interpretation of Quantum Mechanics. Princeton University Press.

Egan, G. (2000). Isolation. Edition Denoël. (Trad. de Quarantine (1992). HarperPrism.)

Everett III, H. (1957). On the foundations of quantum mechanics [thèse de doctorat]. Princeton University.

Feynman, R. (1979). Le cours de physique de Feynman - Mécanique quantique. InterEditions.

Ghirardi, G. C., Rimini, A. et Weber, T. (1985). A Model for a Unified Quantum Description of Macroscopic and Microscopic Systems. Dans L. Accardi et al. (dir.), Quantum Probability and Applications. Berlin, Springer.

Ghirardi, G. C., Rimini, A. et Weber, T. (1986). Unified dynamics for microscopic and macroscopic systems. Physical Review D. 34, 470.

Héraud, J. L, Lautesse, P., Ferlin, F. et Chabot, H. (2017). Representing the quantum object through fiction in teaching: ontological contribution of Gamow's narrative as part of an introduction to quantum physics. Science \& Education, 26 (3-4), 299-322. https://doi.org/10.1007/ s11191-017-9890-6

Laloë, F., (2011). Comprenons-nous vraiment la mécanique quantique? EDP Science/CNRS Editions.

Lautesse, P., Vila Valls, A., Ferlin, F., Héraud, J. L. et Chabot, H. (2015). Teaching Quantum Physics in Upper Secondary School in France. Science \& Education, 24(7-8), 937-955. https://doi.org/ 10.1007/s11191-015-9755-9

Le Bellac, M. (2007). Physique quantique. EDP Sciences/CNRS éditions.

Lévy-Leblond, J. M. (1984). Quantique - Rudiments. InterEditions.

Lewis, D. (1973). Counterfactuals. Harvard University Press.

Lewis, D. (1978). Truth in fiction. American Philosophical Quarterly, 15, 37-46. (Réédité en 1983, Philosophical Papers Vol. 1. Oxford University Press.)

Lewis, D. (2007). De la pluralité des mondes. Editions de l'éclat. (Trad. de On the Plurality of Worlds. (1986). Back Blackwell.)

London, F. et Bauer, E. (1939). La théorie de l'observation en mécanique quantique. Dans Exposés de physique générale III. 1-51, Hermann. 
Maudlin, T. (1995). Three Measurement Problems. Topoï, 14, 7-15.

Ministère de l'Enseignement supérieur, de la Recherche et de l'Innovation. (2015). Référentiel de compétences des mentions de licence. http://www.enseignementsup-recherche.gouv.fr/ cid61532/les-referentiels-de-competences-en-licence.html

Ngô, C. et Ngô, H. (1991). Physique quantique : introduction. Masson

Ozcan, O. (2011). What are the students' models about the « spin » and « photon » concepts in modern physics? Procedia - Social on behavioral sciences, 15, 1372-1375.

Ruyant, Q. (2016). Le réalisme structurale face au problème de la mesure. Lato Sensu : Revue de la Société de Philosophie des Sciences, 3(1), 43-51.

Singh, C., Belloni, M. et Christian, W. (2006). Improving students' understanding of quantum mechanics. Physics Today, 59, 43-49.

von Neumann, J. (1932). Mathematische Grundlagen des Quantenmechanik. Springer. (Trad. von Neumann, J. (1947). Les Fondements Mathématiques de la Mécanique Quantique. Union Française, Comité pour l'Expansion du Livre Scientifique.)

Wigner, E. P. (1967). Symmetries and Reflections, Scientific essays of Eugene P. Wigner. Indiana University Press.

Zeh, H. D. (1970). On the interpretation of measurement in Quantum Theory., Foundations of Physics, 1, 69-76.

\section{NOTES}

1. L'hypothèse fondamentale de cette interprétation est que la fonction d'onde décrit complètement le système. Une discussion sur ce dernier point sera faite dans la sous-section 3.2.

2. L'expérience de Stern et Gerlach, 1922, consiste à observer sur un écran la déviation d'atomes d'argent subissant un gradient de champ magnétique. Contrairement à ce que la mécanique classique prévoit, on observe deux tâches qui révèlent une nouvelle propriété des objets quantiques : le spin.

3. Dans la traduction française du roman, le terme «étalement » est utilisé pour exprimer la notion de « superposition".

4. Ceci est lié au fait que l'atome a une demi-vie d'une heure et qu'on ouvre la boîte au bout d'une heure. (p. 174).

\section{ABSTRACTS}

In higher education in France, in the third year of the physics degree course, the teaching of quantum mechanics is essentially quantitative and formalism is presented as part of the standard interpretation. Moreover, some universities also offer an introduction to quantum mechanics in the second year, including both conceptual and computational teaching. In this article we propose to introduce an epistemological investigation of the physical concepts operating within quantum mechanics, at second-year level, by taking an interest in the delicate so-called 
measurement problem. We hypothesize that lecturers could approach this investigation via a realistic fiction narrative, G. Egan's novel Quarantine. We draw on a theoretical framework, derived from Lewis' work, to show the relevance of this novel for use in a second-year quantum mechanics course.

Dans l'enseignement supérieur en France, en troisième année de licence de physique, l'enseignement de la mécanique quantique est essentiellement quantitatif et le formalisme est présenté dans le cadre de l'interprétation standard. Par ailleurs, certaines universités proposent également une introduction à la mécanique quantique en deuxième année avec un enseignement davantage conceptuel. Nous proposons dans cet article d'introduire un questionnement épistémologique sur les concepts physiques mis en jeu dans la mécanique quantique, au niveau de la deuxième année, en nous intéressant au délicat problème de la mesure. Nous faisons l'hypothèse que les enseignants pourraient s'emparer de ce questionnement au travers d'un récit de fiction réaliste, le roman Isolation de Greg Egan. Nous employons un cadre théorique, issu des travaux du philosophe David Lewis, en vue de montrer la pertinence de ce roman pour une utilisation dans un cours de mécanique quantique en deuxième année de licence.

\section{INDEX}

Mots-clés: mécanique quantique, enseignement supérieur, problème de la mesure, récit de fiction réaliste, ontologie, épistémologie

\section{AUTHORS}

\section{FABRICE FERLIN}

Université de Lyon, Université Claude Bernard Lyon 1, Villeurbanne, France, Fabrice.ferlin@univlyon1.fr

\section{PHILIPPE LAUTESSE}

Université de Lyon, Université Claude Bernard Lyon 1, Villeurbanne, France, Philippe.lautesse@univ-lyon1.fr

\section{JULIETTE TUAILLON}

Université de Lyon, Université Claude Bernard Lyon 1, Villeurbanne, France, Juliette.tuaillon@univ-lyon1.fr

\section{JEAN-LOUP HÉRAUD}

Université de Lyon, Université Claude Bernard Lyon 1, Villeurbanne, France, Jean-

Loup.heraud@univ-lyon1.fr

\section{LIONEL CHAUSSARD}

Université de Lyon, Université Claude Bernard Lyon 1, Villeurbanne, France.

Lionel.chaussard@univ-lyon1.fr 